\title{
Lapurdum
}

Euskal ikerketen aldizkaria | Revue d'études basques |

Revista de estudios vascos | Basque studies review

$4 \mid 1999$

Numéro IV

\section{Lire et comprendre les textes poétiques de tradition orale : l'exemple de la chanson souletine Atharratze jauregian}

Jean-Baptiste Orpustan

\section{(2) OpenEdition}

Journals

Édition électronique

URL : http://journals.openedition.org/lapurdum/1540

DOI : 10.4000/lapurdum. 1540

ISSN : 1965-0655

Éditeur

IKER

Édition imprimée

Date de publication : 1 octobre 1999

Pagination : $57-79$

ISBN : 2-84127-156-0

ISSN : $1273-3830$

Référence électronique

Jean-Baptiste Orpustan, «Lire et comprendre les textes poétiques de tradition orale : l'exemple de la chanson souletine Atharratze jauregian », Lapurdum [En ligne], 4 | 1999, mis en ligne le 01 avril 2010, consulté le 01 février 2020. URL : http://journals.openedition.org/lapurdum/1540; DOI : 10.4000/ lapurdum. 1540 


\title{
Jean-Baptiste ORPUSTAN
}

\author{
LIRE ET COMPRENDRE LES TEXTES \\ POÉTIQUES DE TRADITION ORALE : \\ L'EXEMPLE DE LA CHANSON SOULETINE \\ ATHARRATZE JAUREGIAN
}

Les textes anciens transmis par la tradition orale s'altèrent et se transforment nécessairement au cours du temps, à tel point que celui dont dispose le lecteur peut se trouver fort différent de celui qui fut composé quelques siècles plus tôt. Mieux, il a été prouvé que même les textes écrits pouvaient se trouver altérés au fil des réécritures, des éditions et des publications ${ }^{1}$. Et pour s'en tenir à un des textes par définition "classiques" de la littérature basque, le recueil poétique de Dechepare, premier livre de langue basque à avoir été imprimé, comme le dit son titre de Linguae Vasconum Primitiae, chez Millanges le grand éditeur bordelais en 1545 , tout lecteur ayant pratiqué ce texte fameux, sait qu'il contient des imperfections ou irrégularités de divers ordres, prosodiques bien sûr et linguistiques; et qu'il n'y a peut-être pas lieu de les mettre toutes au compte de l'insuffisance personnelle avouée par l'auteur. Car il y eut sans aucun doute quelques relais et médiateurs, et l'un au moins bien connu, l'avocat Lehet installé à Bordeaux comme d'autres Basques de ce temps-là ${ }^{2}$, que l'auteur remercie explicitement pour son rôle dans l'édition de l'ouvrage. Faute du manuscrit, il sera impossible de mesurer les modifications subies par le texte initial. Mais il est certain, pour prendre un seul exemple, que la Sautrela ou "Saltarelle" qui fait l'avant-dernier numéro, en vieux tercet inégal, pourrait être ajustée sans trop de risque d'erreur, pour faire la rime unique attendue :

assez difficilement à la strophe 2 :

(Berce gendec uste futen

Ecin scriba çayteyen

Oray dute phorogatu Enganatu cirela), où la rime en -en demande inversion d'hémistiche et transformation du participe perfectif enganatu en imperfectif enganatcen du reste plus conforme au sens ("ils se trompaient" est plus exact que le prétérit "ils se trompèrent") et ajout d'une syllabe pour éviter le hiatus ou la synalèphe (à quoi du reste l'auteur est peu attentif en général) : oray dute phorogatu cirela (ou ciraiela en faisant la synalèphe) enganatcen "maintenant ils ont fait la preuve qu'ils se trompaient";

aisément à la strophe 5 (même rime) :

(Bascoac oroc preciatzen

Heuscara ez iaquin harren

Oroc icassiren dute Oray cer den heuscara) où il suffit d'intervertir les termes du second hémistiche du troisième vers : oray heuscara cer den. 
Lorsque le texte a été transmis de longue date et sur plusieurs siècles, par la seule voie orale éventuellement relayée par une ou plusieurs versions écrites éphémères et perdues, avant la collecte écrite qui en fixe au moins approximativement la lettre, comme pour la célèbre Chanson de Berterretche née d'un fait divers politique du milieu du $X V^{e}$ siècle et publiée - sinon recueillie - plus de quatre siècles après dans le recueil de Sallaberry (1870), les altérations subies doivent être à la mesure du temps passé. Le commentateur doit s'attendre à une rude tâche s'il souhaite, comme c'est légitime et même nécessaire, mesurer approximativement les altérations et les insuffisances, formelles et de fond, de la version recueillie. Le texte commenté ici vient de moins loin que la Chanson..., puisque les faits historiques (mais dans quelle mesure le sont-ils?...) en cause, du moins dans l'une des versions, ne remonteraient qu'à la seconde moitié du $\mathrm{XVI}{ }^{e}$ siècle, et il est moins long.

Après le rappel des quatre versions principales de Atharratze jauregian, parmi la vingtaine aujourd'hui connue, celle de Chaho non publiée et de peu antérieure à celle que Francisque-Michel publia dans son ouvrage sur le Pays basque de 1857, celle du recueil de Sallaberry (1870) faisant allusion aux deux précédentes, enfin la version assez différente de J. de Jaurgain (1899) à incipit Ozaze jaurgainian, les éléments d'analyse et de commentaire succinct porteront sur les points suivants :

1) le titre, le thème et les événements relatés;

2) la conduite du récit poétique et l'ordre des strophes;

3) les questions linguistiques;

4) les problèmes de prosodie et versification.

\title{
1. Les quatre versions principales ${ }^{3}$
}

A. Première version du Chansonnier d'Augustin Chaho, non publiée, mais connue des éditeurs suivants, titre particulier apparemment dû à Chaho luimême; les 6 strophes de quatrains monorimes sont alignées en huitains d'hémistiches (ici remis en quatrains, comme dans une autre version du même auteur, mais avec la majuscule à l'initiale d'hémistiche conservée), avec quelques variantes de détail dues sans doute à Chaho lui-même essayant de retrouver le rythme de la prosodie primitive pour le compte des syllabes. L'orthographe suit la prononciation souletine à laquelle Chaho est particulièrement attaché, distinguant notamment les voyelles $u$ et $o u$ comme en graphie française. Pour la clarté du récit, des guillemets et tirets entre parenthèses sont ajoutés dans les parties dialoguées :

\author{
Santa Clara \\ I \\ Atharratze jaureguian Bi citroiñ loratu. \\ Hongariaco erreguec Batño du galthatu. \\ Arraposta ukhen du Eztirela hountu, \\ Hountu diratinian Batño ukhenendu. \\ II \\ (“-) Aita saldu naizu Ardi bat* bezala, \\ Bai eta desterratu Espanintzan bezala** \\ Ama bizi ukhen banu Aita zu bezala, \\ Ni ezcunturen nintzan* Atharratze Salala.
}


*Variantes : Anchu bat, Achuri bat, Bigabat

** Var. : Hongarian behera

*** Var. : Nintzan ni ezcunturen

III

("-) Ahizpa jaunts ezazu Saya berdia.

Nic ere jauntsiren dut Satina churia

Ingoiti hor heltu da Zure senhar gueya

Botzez utzi ezazu Zure sorth'etchia.

IV

("-) Aita johanen gira Oro algarrekin,

Etcherat jinen cira Changrin handirekin,

Bihotza tristeturik Beguiac bustiric,

Eta zure alhaba Thumban ezarriric.

$\checkmark$

(")Ahizpa zuaza ora Salako leihora,

Iphar ala heguoua Cer den jaquitera.

Ipharra balinbada Goraintzi Salari

Et'aldiz ene tcherca Jin dadila sarri. (")

VI

Atharratzeco ceñiac Tristeric du joiten*,

Andere Santa-Clara Bihar da phartitzen**

Haren-peco zaldia Urhez da zelatzen***

Hanco tchipi handiac Belzez **** dira bestitzen*****.

Var. : * Berac arrapicatzen

** Herritic phartitzen

*** Da chouriz estaltzen. **** Belchez.

***** Dira bertzez beztitzen

B. Version de Francisque-Michel ${ }^{4}$.

Annoncée comme une "véritable romance du vieux temps" et "une belle ballade en dialecte souletin [...] très goûtée dans le pays et jusque dans le Labourd", elle a été recueillie par l'auteur dans les Instructions relatives aux poésies populaires de la France, ouvrage publié sous un titre un peu différent à la Bibliothèque Impériale en 1853 (la date en chiffres romains portée à la note 1 . p. $265 M D C C$ LIII qui correspond à 1753 est une erreur, recopiée ensuite par tous les commentateurs). Elle se compose des mêmes 6 strophes que la version de Chaho et dans le même ordre. A part la graphie et la phonétique et des éléments de morphologie et de lexique labourdins, justifiés par la référence au succès dans cette province, et quelques coquilles inévitables (Atharratceco... au titre et Atharrats... au premier vers etc.), elle ne contient que peu de variations dans le texte lui-même, que l'on se contente de rappeler vers après vers devant le texte de Chaho entre parenthèses:

Atharratzeco ezcongaia

$I$

... doratu - (loratu)

Ongriagaray horrec bat du galdatu - (Hongariaco erreguec batño...)

Errepostu içan du - (Arraposta ukhen du...)

ontcen direnian - (Hountu diratinian...) 
II

... saldu nauçu miga bat - (biga bat... parmi les variantes de Chaho)

... oi! Españara - (Espanintzan bezala/Hongarian behera)

Ez nintçan ezconduren Atharrats Salara.

(Vers de sens contraire chez Chaho : Ni ezcunturen nintçan...)

III

... erroba pherdia - (Saya berdia)

... cure jaun* gueia - (zure senhar gueya)

* Francisque-Michel s'est rendu compte ici - mais pas partout, ou il n'a pas osé intervenir - que le vers était faux et a mis en note, ajoutant une syllabe et rejoignant le texte de Chaho (qui a aussi des vers faux ailleurs) qu'il connaissait peut-être : "Ne faudrait-il pas plutôt senhar?". Notons que, depuis Chaho, c'est la sœur qui se met en blanc et la fiancée en... vert, sauf dans la version D.

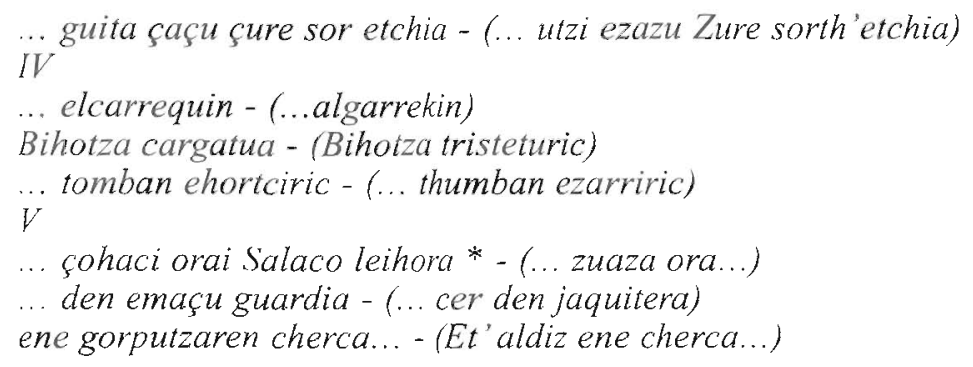

*La traduction de Francisque-Michel, qui est souvent approximative ou même fautive dans son ouvrage, est ici : "... allez vers la fenêtre de Salles", ce dernier mot traduisant la maison "Sala" de Tardets, alors que le texte dit clairement qu'il s'agit de la "salle" (nom commun) de la maison de la fiancée qu'elle va quitter. Sallaberry reprend le même sens mais dans une formule maladroite quoique malheureusement commune en "français" du Pays basque : "à la fenêtre de chez Sala"!

\section{$V I$}

... ezquilec bere motuz joten - (... ceñiac Tristeric du joiten

Hango chipi handiac beltchez dira beztitzen.

... Berac arrapicatzen)

Les demiers vers sont très proches du texte de Chaho, mais sans les variantes que celui-ci introduisait (da chouriz estaltzen, dita bertzcz beztiizen).

\section{Version de Sallaberry $(1870)^{5}$.}

Agrémentée d'un bref commentaire, qui s'arrête moins sur les difficultés historiques posées par le "roi de Hongrie" de Chaho (le conditionnel avoue toutefois un certain scepticisme : "il s'agirait d'un roi de Hongrie") que sur la difficulté "onomastique" de l'Ongriagaray du "savant professeur", nom dont Sallaberry déclare à très juste titre "il n'existe pas et il n'est pas vraisemblable qu'il ait existé", la chanson s'intitule Atharratze jauregian (que le premier vers, pour raison à la fois de métrique et de musique, puisque la mélodie notée précède le texte, rectifie en Atharratz jauregian), s'allonge ici presque du double et atteint 10 strophes de quatrains, de nouveau en dialecte souletin (introduction du tréma pour la voyelle arrondie $\ddot{u}$ propre à ce dialecte). 
Voici d'abord les 4 strophes en plus :

Strophe $n^{\circ} 2$ introduite entre les strophes I et II de Chaho, comportant la description topographique de Tardets :

2

Atharratzeko hiria hiri ordoki,

Hur handi bat badizu alde bateti;

Errege bidia erdi erditi,

Maria-Madalena beste aldeti.

Strophes $n^{\circ} 4$ et $n^{\circ} 5$ introduites entre les strophes II (reproche de la fille au père pour l'avoir mariée contre son gré) et III (la sœur de la fiancée l'invite à se vêtir) de Chaho; la strophe $\mathrm{n}^{\circ} 4$ fait encore parler la fiancée s"adressant à sâ sœur, mais avec un vers pris dans la strophe III de Chaho et qui sera répété au n ${ }^{\circ} 6$; la strophe $n^{\circ} 5$ est la réponse de la sœur, contenant donc un vers repris à la précédente et puis répété, rappelant le style fâcheux des airs d'opéra;

4

("-) Ahizpa, juan zite portaliala,

Ingoiti horra düzü Ongriako Erregia;

Hari erran izozü ni eri nizala,

Zazpi urthe huntan ohian nizala.

5

("-) Ahizpa, enükezü ez sinhetsia,

Zazpi urthe huntan ohian zirela;

Zazpi urthe huntan ohian zirela;

Bera nahi dükezü jin zü zien lekhila.

Entre la strophe $\mathrm{n}^{\circ} 6$ (III de Chaho: invitation à s'habiller), et la $\mathrm{n}^{\circ} 8$ (qui est la IV de Chaho: annonce du départ du cortège) et adressée au père dans le même reproche que la strophe $n^{\circ} 3$ (II de ('haho), la strophe $n^{\circ} 7$ est manifestement transportée d'une chanson navarraise ancienne rapportée par Oyhénart en 1665, avec quelques changements (voir ci-dessous) :

\section{7}

(")-) Aita, zü izan zira ene saltzale,

Anaie gehiena dihariren harzale,

Anaie artekua zamariz igaraile,

Anaie chipiena ene lagüntzale.

Variantes de détail dans les strophes identiques:

I (I de Chaho)

... doratü (Chaho avait déjà mis ce mot au lieu du loratu de son premier texte, plus logique et meilleur linguistiquement comme emprunt latin ancien, mais qui ne reparait plus dans les versions principales).

Ongriako Erregek (le Hongariaco erreguec de Chaho meilleur phonétiquement pour le groupe consonantique, mais exigeant une synalèphe co-Er pour garder le mètre de treize syllabes $7+6$ qui est la base prosodique de toutes les versions, était sans doute une correction "savante" de Chaho : le mot hong(a)ria fait de toutes façons difficulté pour la métrique). 


\section{3 (II de Chaho)}

idi bat bezala (les comparaisons de Chaho "brebis, brebis stérile, agneau, génisse" comme celle de F.-M. "génisse", étaient pourtant plus adéquates au personnage de la fille mal mariée que celle du "bœuf")...

... Ongrian behera (cf. Chaho : Hongarian behera avec une syllabe de trop)...

Bena bai ezkuntüren Atharratze Salala (sens de la version de Chaho, mais avec une syllabe de trop comme elle : 7 au lieu des 6 requises).

\section{6 (III de Chaho)}

Ahizpa jaunts ezazü arrauba berdia (proche de Chaho, mais avec arrauba comme F.-M. erroba, au lieu de zaya, le mètre 7+6 est juste)

... dit ene churia (le mot satina des deux autres est écarté)

Ingoiti horra düzü Ongriako Erregia (les formules de Chaho et F.-M. sont corrigées en tournure souletine pour horra düzü et par le titre royal pourtant moins adéquat que senhar gueya y compris prosodiquement, puisqu'il faut ici compter les mêmes mots différemment de la strophe 1).

\section{7 (IV de Chaho)}

\section{Bihotza kargaturik... (comme F.-M.)}

... thumban ehortzirik (idem).

\section{9 ( $V$ de Chaho)}

... zuza orai (zuza est une coquille pour zuaza dissyllabique avec synérèse normale, et orai remplace ora de la seule version en faux octosyllabes de Chaho sans doute "corrigé" par lui-même pour la rime, inutile puisque c'est en réalité la césure d'hémistiche)...

... denez jakitera (proche de Chaho cer den, bien meilleur pour le sens que F.M. emaçu guardia)...

Ene khorpitzaren cherca (comme F.-M. mais en souletin : le "corps" explicite tragiquement ce qui dans Chaho ene tcherca restait implicite)...

\section{0 (VI de Chaho)}

Atharratzeko zeñiak berak arrapikatzen (seconde variante de Chaho mais c'est la première qui était prosodiquement juste: Tristeric du joiten):

Hanko jente gazteriak beltzez beztitzen,

Andere Santa Klara hantik phartitzen;

Haren peko zamaria ürhez da zelatzen.

Cette strophe est assez considérablement transformée par rapport aux versions précédentes : interversion des vers pour mettre à la fin le départ de la fiancée sur le cheval "sellé d'or", alors que les autres s'achevaient sur les images de deuil, on peut le noter, plus conformes à la tonalité tragique; zaldia est remplacé par zamaria mot plus rare et sans doute tenu pour plus noble, mais la métrique est faussée (8 au lieu de 7), de même qu'au vers 2 dans la formule nouvelle hanko jente gazteria ( 8 au lieu de 7), et si l'ensemble du vers 2 fait bien 13 syllabes c'est au détriment de la césure qui doit être régulière dans ce type de vers; gazteria "jeunesse" remplace chipi handiac "grands et petits" qui était probablement la version d'origine (il s'agit à n'en pas douter des rangs sociaux et non des "jeunes et vieux"); de même pour bihar da, un départ du "lendemain" qui doit évoquer le départ... pour l'enterrement de la fiancée (voir plus loin : l'histoire et la légende), remplacé par le banal hantik. 


\section{La version "Ozaze Jaurgaiñian" 6}

Dans cette version qui remplace parfois les précédentes, sous le titre Atharratzeko ezkongaia identique à la version de Francisque-Michel, l'histoire change partiellement : plus de roi de Hongrie, ni d'improbable "Ongriagaray", mais une fille de la maison noble Jaurgain d'Ossas (deuxième maison noble du lieu après le domec du podestat) que l'on marie au seigneur de la Salle de Tardets, ce qui correspond à une réalité historique, puisque Charles de Luxe en même temps seigneur de Tardets ennemi têtu des protestants et de la reine de Navarre et dame de Béarn Jeanne d'Albret avait épousé en secondes noces (un premier mariage en 1564) en 1584, âgé de 48 ans, une demoiselle de Jaurgain âgée de 17. C'est l'historien Jean de Jaurgain qui transforme la chanson, déjà fort altérée au cours des âges, pour y plaquer, non sans inconséquences diverses, ce mariage du $X V I^{e}$ siècle qui faisait honneur à sa propre famille. Elle diffère principalement des précédentes non seulement par le titre, la localisation, le motif historique, des éléments du dialogue, mais aussi par un ordre différent des 9 strophes qui la composent, la strophe 7 de Sallaberry (transfert de la chanson navarraise) étant absente, et l'orthographe passablement modifiée par rapport au dialecte souletin (élimination des consonnes aspirées etc.), avec un luxe de ponctuation peu conforme à la tradition ancienne. En voici strophe par strophe les correspondances avec les versions précédentes, celle de Sallaberry d'abord, de Chaho et Francisque-Michel ensuite, et les variantes notables:

I

Ozaze Jaurgaiñian bi zitroiñ doratï,

Atharratzeko jaonak bata dü galdatü.

Uken dü arrapostü eztirela huntü;

huntürik direnian batto ükenen dü

La modification décisive qui change le sens du récit porte sur les noms de lieu : les "citrons dorés" exotiques répétés depuis la seconde version de Chaho et Francisque-Michel, mais en contradiction avec le texte, puisqu'ils ne sont pas encore "mûris", c'est-à-dire précisément "dorés", sont les filles de la maison Jaurgain d'Ossas, et l'épouseur le seigneur de la "salle" de Tardets. Dans ce cadre, le récit est cohérent jusqu'au bout. L'autre modification est prosodique : le mètre de 13 syllabes avec césure régulière $7+6$ conformément à la tradition du vers basque, mais sans élision ni synalèphe régulière, est mieux observé surtout dans les premières strophes, ce qui suppose à n'en pas douter une correction des versions précédentes par le collecteur ou l'éditeur, quoique la justesse prosodique ne soit pas conservée jusqu'au bout.

Correspondance strophe à strophe avec les autres versions :

$I=$ strophe I des autres versions;

Ozaze Jaurgaiñian bi zitroin...

$2=$ strophe 4 de Sallaberry absente des autres versions:

(")-) Portaliala juan zite, ahizpa maitia

Ingoiti horra düzü Atharratzeko jaona..

$3=$ strophe 5 de Sallaberry absente des autres versions, le vers 2 n'est plus répété au troisième, sans pour autant quitter le style "opéra":

(“-) Bai, bena enükezü hortan sinetsia,

Hari erraiten badot zü eri zirela, 
Zazpi egün hoietan ohian zirela,

Bera nahi dükezü jin zü ziren lekila.

4 = strophe III de Chaho et F.-M., 6 de Sallaberry, mais c'est la sœur qui continue à parler à la fiancée et non celle-ci comme dans les autres, et la fiancée qui est invitée à se vêtir de blanc (selon les usages modernes), par sa sour qui se met en vert:

(“) Ahizpa, jauntz ezazü arrauba xuria,

Nik ere jauntziren dit ene zaia berdia,

Ingoitik horra...

5 = strophe V de Chaho et F.-M., 9 de Sallaberry, le prénom Klara "Claire" (et non "Sainte-Claire" qui est normalement un hagionyme nom de lieu ou de maison, quoique inconnu dans la région) est donné à la sœur; le jeu des minuscule/majuscule permet d'opposer la "salle" de la maison de la fiancée à la "Salle" de Tardets maison du fiancé; seul le dernier vers est faux $(8+7)$ :

(“-) Klara, zuaza orai salako leihora,

Iparr' ala hegua denez jakitera ;

Iparra balinbada, goraintzi Salari,

Ene korpitzaren xerka jin dadila sarri.

$6=$ strophe IV de Chaho et F.-M., 7 de Sallaberry, mais la fiancée fait des reproches à sa mère, en contradiction formelle avec les autres textes où elle les fait à son père, et sans doute aussi (à moins de circonstances familiales et historiques particulières dans la maison Jaurgain) avec la tradition sociale ancienne bien affirmée dans la littérature, basque ou autre (dans la chanson navarraise citée plus haut le reproche va au père et à la mère) :

("-) Ama, juanen gira oro alkarreki,

... zure alaba tumban ehortzirik.

7 = strophe VI et dernière de Chaho et F.-M., 10 et dernière de Sallaberry, qui fait entendre cette fois la cloche (le glas) d'Ossas et non de Tardets :

Ozazeko zeñiak dii uriapikatzen,

Jaurgaiñek'anderia herritik partitzen;

Haren peko zaldia ürrez da zelatzen,

Hanko txipi handiak beltzez dira beztitzen.

$8=$ strophe 2 de Sallaberry reprise telle quelle (le site de Tardets, où la fiancée est censée se rendre en cheval sellé d'or), absente chez Chaho et F.-M.

$9=$ strophe II de Chaho et F.-M., 3 de Sallaberry, reproches à la mère au lieu du père, la situation est inversée, et c'est le père, mort, qui aurait empêché le mariage de sa fille et son exil en Espagne :

(“-) Ama, saldü naizü biga bat bezala,

Bai eta desterratü, oi! Españiala.

Aita bizi ükhen banü, ama, zü bezala,

Enündüzün ezkontüren Atarratze salala.

\section{Histoires et légendes mêlées.}

Ce poème ne se référant au XVI ${ }^{\circ}$ siècle finissant, pour les éléments historiques repérables, que dans la variante introduite (inventée?) par Jaurgain, a été défini par Francisque-Michel, très anachroniquement, comme une "ballade" au sens 
romantique du mot (et il ne rappelle en rien, en effet, dans sa forme imparfaite et son style improvisé, la vraie ballade médiévale, poème strophique à forme fixe). C'est exactement un "récit poétique en vers", en basque eresia selon la plus ancienne et la meilleure appellation donnée par Oyhénart (1657), qui en connaissait peut-être une version. A s'en tenir au récit, c'est-à-dire à la succession des faits rapportés, des personnages cités et de leurs relations, des lieux évoqués, trois histoires, en partie contradictoires, s'y mèlent :

$1^{\circ}$ Un mariage avec un roi de Hongrie, mariage sans aucun doute mythique $\mathrm{e}^{t}$ légendaire, arrangé pour l'une de ses deux filles par le seigneur de la Salle de Tardets, Atharratze jauregia dans la chanson, maison parfaitement historique : depuis la fin du XIV e siècle le seigneur de la Salle de Tardets était seigneur de Luxe, par le mariage, contracté vers 1365 selon Jaurgain ", de Saora ou "Saurine" héritière et dame de Luxe, Ostabat et Lantabat, la seconde fille d'Arnaud-Loup II de Luxe, "grand chambellan et favori de Charles le Mauvais" roi de Navarre (l'aînée avait épousé Rodrigo d'Uriz, richomme de Navarre, gouverneur des châteaux de Tudela et Caparroso, mérin de la Ribera et de Tudela etc. qui se disait aussi "seigneur de Luxe" par ce mariage du vivant de sa femme), avec Arnaud-Sanche IV seigneur de la Salle de Tardets, Ahaxe en Cize etc., qui signait arnaut Santz de tardetz seynor de Luxe. C'est lui qui conclut dans l'église Notre-Dame du bout du pont à Saint-Jean-Pied-de-Port le 2 avril 1384 en présence du roi et de l'infant de Navarre, Charles II et le futur Charles III, au nom de sa femme Saura et de ses successeurs, la paix avec le seigneur et le lignage de Gramont.

$\mathrm{Au}$ début du $X V \mathrm{I}^{\circ}$ siècle au moment de la guerre de Navarre (1512-1530) commencée par Ferdinand d'Aragon et poursuivie sous Charles-Quint, Jean IV seigneur de Luxe, Tardets etc. avait remis le château fort de Luxe en Mixe à Henri II d'Albret roi de Navarre en 1515, et s'était installé à Tardets. Puis, pour une nouvelle trahison, le roi de Navarre lui avait confisqué tous ses biens mixains en 1524 et fait raser peu après le château fort. Lorsque Jeanne d'Albret fille de Henri II d'Albret devint protestante en 1560 et que ses États, royaume navarrais (basnavarrais seulement depuis 1530) compris, furent mis à l'encan par décision papale, c'est Charles de Luxe le fils de Jean IV mort en 1559 qui mena avec des seigneurs souletins et bas-navarrais (Domezain, Armendaritz, Antonin vicomte de Baïgorry-Etchauz etc.) la rébellion contre la reine de Navarre dans les années 1567-1570. Les troupes de Jeanne d'Albret ne reprirent définitivement le pays qu'en août 1570 à la paix dite "de Saint-Germain". Les révoltés demandèrent le pardon de la reine, qui fut d'abord refusé à Luxe comme meneur de la révolte et stipendié par le roi de France : il dut s'exiler à Ochagavia en Navarre et ne revint en France qu'après la mort de la reine en 1572. Ses menées anti-protestantes ne s'arrêtèrent pas pour autant, et on lui attribue diverses exactions dont le meurtre d'Eneco de Sponde autrefois secrétaire de Jeanne d'Albret (et père du poète Jean de Sponde et du futur évêque de Pamiers Henri) dans l'église de Saint-Palais en 1594.

Il était en relation avec le roi d'Espagne qui soutenait les ultra-catholiques de la Ligue contre Henri de Navarre devenu roi de France à la mort de Henri III (1589) et tout récemment converti au catholicisme (1593) : c'est ce que révèle une lettre en basque qu'il fait écrire sans doute de Tardets à l'une de ses filles, 5 de son premier mariage dont l'aînée Charlotte-Catherine se marie en 1593 à Henri duc de Montmorency (1534-1614) et gouverneur du Languedoc, 4 (et 2 fils 
dont l'un fut "seigneur de Jaurgain" la maison de sa mère) du second sans doute encore un peu jeunes : datée du 30 mars 1595, elle est adressée au régent de Navarre pour le roi d'Espagne à qui elle parvint, via Ochagavia, le 12 juillet, et donne des informations (en grande partie fausses) sur la situation des armées de Henri IV contre celles du duc de Mayenne ${ }^{8}$.

$2^{\circ} \mathrm{La}$ version $\mathrm{D}$ racontant le mariage d'une demoiselle de Jaurgain d'Ossas avec le seigneur de la Salle de Tardets ferait allusion à ce Charles de Luxe, son exil à Ochagavia étant des années 1570-1572 : il faut supposer que le mariage était fait à cette date, et que c'est de son exil en compagnie de son mari que l'héroïne se plaint, mais le second mariage étant postérieur (1584), c'est impossible, à moins qu'il n'ait été à nouveau exilé. Cet exil en Espagne est cité dans toutes les versions sauf la première de Chaho : dans celle-ci le vers Bai eta desterratu Ezpanintzan bezala est un quasi non-sens ("et exilée comme (si) je n'étais pas"?). La seconde version de Chaho, qui introduit aussi Ongriagarai horrek, dit España barnara "au cœur, au fond de l'Espagne", ce qui la rapproche de celle de Francisque-Michel (1857) : celle-ci porte, avec une exclamation qui fait cheville, oi! Españara ("exilée, hélas! en Espagne") comme la version D; mais c'est Chaho qui ajoute d'abord en marge une variante (de son propre chef?) Hongarian behera "au fond de la Hongrie" que reprend la version C de Sallaberry.

Manifestement deux "histoires" se croisent, le mariage, réel ou imaginaire, romantique et tragique en tout cas, d'une fille noble de la "Salle" de Tardets très loin de chez elle, et celui plus terre à terre d'une fille de Jaurgain à la "Salle" de Tardets, réel mais incompatible avec la chronologie. Chacun s'ingénie à les rendre cohérentes: Chaho avec sa variante reprise dans la version Sallaberry (Ongrian behera), le même Chaho suivi par Francisque-Michel (ou son informateur) en fabriquant le nom Ongriagaray qui évite d'envoyer la fille de Tardets en Hongrie, la version D, la plus cohérente en apparence mais historiquement impossible, en décrivant le départ de la fiancée d'Ossas à... Tardets, ce qui n'est pas bien loin, et quitte ensuite à lui faire suivre son mari dans son exil d'Espagne, thème introduit seulement dans la neuvième et dernière strophe, ce qui est également cohérent dans le récit : c'est de là, de son exil d'Espagne, que se plaint l'exfíancée. Alors le récit poétique ne serait qu'un exemple de plus du "mariage arrangé" ou de la "mal mariée, mariée contre son gré", topique de la poésie ancienne en Pays basque et ailleurs, et le contexte tragique inscrit pourtant tout au long de la chanson serait très affaibli, et même gommé.

$3^{\circ}$ La seconde version de Chaho reprise par Francisque-Michel dit une histoire intermédiaire : il s'agit toujours d'une fille de la Salle de Tardets, mais elle est demandée par un Ongriagaray qui mêle dans son nom la Hongrie de la lígende sans doute primitive, et la toponymie basque habituelle : monstre onomastique à deux têtes. Et elle est bien exilée... en Espagne. Y a-t-il allusion à un autre mariage, une fille de la Salle de Tardets mariée en Espagne? L'histoire, ancienne mais aussi moderne, doit en avoir connu plus d'un exemple, et le récit reste parfaitement, lié ou non à une réalité et un fait particuliers, dans l'ordre du vraisemblable.

$4^{\circ}$ Une autre histoire, seulement de tradition parlée, vient apporter d'autres informations et compléter le récit ressenti, dans toutes ses versions après Chaho, comme lacunaire et tronqué, volontairement par l'improvisateur ou par oubli, de quelques péripéties attendues et donc, après Chaho, "rallongé" par les uns et les autres avec plus ou moins de bonheur. Qu'est-il arrivé? le mariage a-t-il été 
conclu? a-t-il été heureux? que signifient des éléments de narration comme le symbolisme des couleurs, le tocsin qui se met à sonner tout seul, la population tout entière qui se vêt de noir?...

Quand les enfants n'accédaient à la culture littéraire basque qu'en apprenant les chansons à la maison et en les chantant (en 1943 avait été publié le recueil Kantuz des abbés Lafitte et Etchemendy avec 120 chansons où Atharratze dans la version Sallaberry est classé au n ${ }^{\circ} 42$ parmi les "chansons touchantes" Kantuhunkigarriak, et non les chansons d'amour Amodiozko kantuak), le sens de cette chanson intriguait. Il me souvient d'avoir entendu donner à notre mère interrogée à ce sujet l'explication suivante : la fiancée, ayant sans doute appris que c'est le vent du nord qui soufflait, s'était jetée par la fenêtre de sa "salle", celle-ci située haut à l'étage comme dans toutes les maisons-tours de facture médiévale qu'étaient les anciennes maisons nobles principales. Ainsi prenaient sens les allusions funèbres ("mis - pour Chaho - ou enterré dans la tombe", glas, habits de deuil), et surtout l'expression "ene khorpitzaren cherca jin dadila sarri", "qu'il vienne tout à l'heure chercher mon corps". Chaho mettait seulement Et' aldiz ene cherca qui avait l'avantage avec son élision à là Oyhénart (et que celui-ci lisait aussi avec raison dans les chansons anciennes) de faire un hémistiche juste (tous les autres sont faux), tout en se contentant d'un sens implicite d'ailleurs sans équivoque : Sainte-Claire dit exactement "Adieu (en fait goraintzi est l'égal de "portez-vous bien", littéralement "Haut dorénavant" : c'est un "Adieu !" et non un "Au revoir!") à Sala, mais qu'il vienne me chercher etc..." L'idée du suicide est donc en conformité exacte avec la dénotation aussi bien qu'avec la connotation funèbre.

Et notre mère ajoutait ceci, qui reprend un thème assez répandu dans la littérature amoureuse de ton dramatique : "on mit le corps dans un cercueil, mais personne ne put le lever sinon "Sala". Or l'ouvrage prétendument daté de 1753 qui aurait contenu la première version du texte comportait un récit en prose recueilli auprès d'une "vieille femme de Biarritz" par le collecteur, "M de Quatrefages" que Francisque-Michel présente implicitement comme un de ses contemporains : on ne donne pas du "Monsieur" aux gens du siècle précédent, et on n'utilise pas en français, pour le passé lointain, le passé composé : "M. de Quatrefages l'y a recueillie, ou plutôt une traduction lui en a été dictée"... Texte curieux et peu utile dans son détail, bourré de fautes de français, commençant par le glas de Tardets en première strophe, mais visiblement désordonné (les citrons "fleuris", comme dans la première version de Chaho, et non "dorés", ne paraissent qu'à la quatrième strophe après les reproches au père etc.), mais s'achevant par le suicide de Sainte-Claire : depuis la "croisée" de sa salle, "elle se précipite et tombe morte", en voyant arriver son futur mari. Mais ce dernier n'est pas le roi de Hongrie absent du texte, il se nomme "Sala". Et bien que seul ce dernier puisse lever son corps ici aussi, le texte reste au fond incohérent.

Cette rencontre sur un point essentiel qui est le suicide de la fiancée, de deux traditions orales, biarrotte et bas-navarraise, à un siècle (mais non deux) de distance, permet de reconstruire le récit poétique primitif avec beaucoup de vraisemblance : une des deux filles de la Salle (dite en basque jauregi) de Tardets (ou d'un autre lieu où il y a une maison noble principale et une autre maison, noble ou non, dite Sala : ainsi par exemple à Sibas qui est en Haute-Soule comme Tardets, à Chéraute, Viodos, Undurein; et à Tardets, à côté de la "Salle" ancien- 
ne avec ses 7 fivatiers ou botoys à Abense-de-Bas, la "ville neuve de Tardets" fondée par le dernier vicomte de Soule en 1299 avait aussi une "salle" citée au Censier à la fin du XIVe siècle : lostau de La sale) est promise au roi de Hongrie; mais elle aime I'héritier de la maison Sala (d'où les deux sens du mot : la "salle" du jauregi ou domec de Tardets ou d'un autre lieu, et la maison "Sala"); elle joue son destin à pile ou face, et plus poétiquement au "vent du nord ou du sud"; c'est le vent du nord ("Adieu à Sala!") et elle se jette par la fenêtre; le glas se met à sonner tout seul, prenant parti miraculeusement pour la demoiselle Sainte-Claire et contre le mariage arrangé, et la population se vêt de deuil; seul Sala peut lever le corps pour l'enterrement annoncé; et ainsi toutes les indications et les propos de Saint-Claire retrouvent leur cohérence.

Sur cette histoire légendaire, appuyée peut-être sur un mariage ou projet de mariage réel d'une héritière de la noblesse principale de Soule (ou même des territoires voisins) avec un prince d'Europe centrale, rendu vraisemblable par les relations des familles royales d'Espagne ou de France avec la cour de Hongrie ou quelque autre événement qui pourrait être déniché dans l'histoire, ancienne à coup sûr, et probablement médiévale", les poètes improvisateurs d'abord, correcteurs ensuite au XIX ${ }^{c}$ siècle ou même plus tôt, ont brodé. La strophe de la chanson médiévale et navarraise Andre Emili ("Dame Émilie est dame haute : elle ne peut ni pétrir le pain ni sarcler le mil... qu'elle s'en aille au pays des Maures..." ${ }^{10}$, y a été abusivement mais logiquement intercalée, puisque le reproche au père marieur et "vendeur" (en réalité aux parents, d'où la mère de la version D) est un topique des chansons de la mal mariée. En fait la chanson d'Émilie, qui évoque les relations des Navarrais avec les Maures, et remonte au moins dans son principe audelà du XIII ${ }^{e}$ siècle, avec la "rançon" encore archaïque des "poids d'or et des barriques de miel", pourrait être l'une des sources lointaines d'où celle-ci, tournée vers un autre "Orient" mais sans quitter l'allusion à l'Espagne, serait issue.

Quant au titre de Chaho Santa Clara censé nommer la fiancée, qui ne saurait être en principe une allusion à sainte Claire compagne mystique de saint François d'Assise au XIII siècle (il y eut un couvent de Clarisses à Bayonne par exemple), contredite par le prénom de la sœur dans la version D qui trouvait sans doute la référence onomastique et religieuse (qui avait plu au contraire à Chaho par son côté inattendu et en un sens fantaisiste) incompréhensible, il reste parfaitement mystérieux sous nos latitudes, où les noms médiévaux de maisons hagionymiques, nobles ou non, ne sont pourtant pas rares. Le Dictionnaire de la noblesse française de E. de Séréville et F. de Saint-Simon " signale bien une famille "Suisse de Sainte Claire", mais en Lorraine et seulement comme noblesse d'Empire, ce qui n'est conforme ni géographiquement ni chronologiquement. Sauf nouvelle information sur une famille ou terre de ce nom existant au Moyen Age et entrée dans une maison noble basque, on peut le tenir pour l'un des éléments légendaires de la chanson, comme le sont aussi les exotiques "citrons dorés", le roi de Hongrie, la robe de satin, le cheval scellé d'or, le glas qui sonne tout seul etc. On n'excluera pas pour autant que ce soit simplement au départ un prénom féminin porté dans les familles nobles de la région (par exemple "Claire de Gramont" qui reconstruisit en 1530 le château de Bidache endommagé par les troupes de Charles-Quint au cours de la guerre de Navarre en 1523) : c'est la solution retenue par la version $\mathrm{D}$, mais en donnant ce prénom à la sœur de la fiancée. 


\section{La conduite du récit et l'ordre des strophes}

La version courte de Chaho et Francisque-Michel, la plus ancienne sauf nouvelle découverte, posait le moins de problèmes dans l'ordre des strophes : 1. thème du mariage projeté ; 2 . (Au matin du départ) reproches de la fiancée à son père qui l'a "vendue", regret de la mère déjà défunte qui aurait empêché le mariage (complicité de mère-fille aussi fréquente que son contraire : voir le théâtre de Molière par exemple); 3. la sœur de la fiancée l'invite à s'habiller et à partir "avec joie" botzez (les autres mettent botzik "joyeuse") au devant de son promis; 4. la fiancée s'adresse à nouveau à son père pour lui annoncer qu'il sera triste de l'avoir (réellement ou symboliquement) "mise à la tombe"; 5. elle s'adresse ensuite à sa sœur pour aller voir "à la fenêtre de la salle" (de la maison) quel vent il fait : s'il fait vent du nord "adieu à Sala" (son promis ou son bienaimé ?); 6. le glas sonne, la population se vêt de noir pour le "départ", sur son cheval "sellé d'or" (mais la variante de Chaho dit "couvert de blanc"...), de "damoiselle Sainte Claire"... Et c'est tout. Avec toutes ses lacunes, habituelles et même normales dans les poèmes transmis oralement de loin, qui rendent les propos plus souvent allusifs que clairs, ce qui n'est pas sans doute un trait spécifique des chansons anciennes parce qu'elles sont anciennes, mais davantage pour le moins parce qu'elles sont transmises oralement de loin, donc lacunaires dans la suite des péripéties du récit, le récit reste cohérent dans ses grandes lignes, s'il est bien obscur dans plusieurs détails (d'où, en particulier, les variantes mises en marge par Chaho).

Les auteurs et chansonniers vont s'efforcer de combler quelques vides, comme on le voit dans Sallaberry et la version Ozaze. Le premier ajout est la strophe décrivant le site de Tardets, qui n'a pourtant aucun lien explicite avec le récit. Tout au plus peut-on imaginer qu'elle informe l'auditeur (en verbe allocutif voussoyé : badizü) comme le ferait un guide touristique : "La ville de Tardets est sise sur un plateau : sur un côté une grande rivière, en plein milieu passe le chemin royal (la grand-route), de l'autre côté Marie-Madeleine" (la chapelle de la Madeleine, dite en Basse-Navarre "La Récluse"). La description, malgré le "plateau" sens exact du composé médiéval ordoki, est un peu avantageuse pour un site bien escarpé, mais peut connoter plusieurs éléments du récit : la grandeur du mariage et de la "Salle" de Tardets d'un côté, une double dimension tragique de l'autre, la rivière si souvent lieu de naufrage (ou de suicide) et la chapelle de la "Récluse" la repentie d'amour... Connotation seulement : car dans un autre contexte tout ceci prendrait un autre sens. Et c'est ce qui fait penser que cette strophe, que Chaho fils de Tardets aurait sûrement inscrite s'il l'avait sue, est probablement rajoutée, et tardivement; tous ses vers sont du reste diversement faux : $8-5,7-5,6-5,7-5$ (sans aucune élision ni synalèphe) chez Sallaberry et la version D. Mais si Sallaberry la met tout de suite après la première strophe annonçant le mariage, D la met en avant-dernier après le "glas d'Ossas" et l'habillement en noir des "grands et petits", ce qui est bien surprenant.

Disons tout de suite que les reproches à la mère que la version $\mathrm{D}$ met en neuvième et dernière strophe sont dans une position encore plus absurde, que la fiancée soit morte, ce que cette version semble vouloir exclure malgré les diverses connotations tragiques et en conformité avec le mariage supposé réel de la fille d'Ossas avec Luxe-Tardets, ou qu'elle soit partie pour se marier sur son cheval sellé d'or. 
Mais pour revenir d'abord à Sallaberry, après l'insertion de la description de Tardets, le reste de son récit suit les lignes générales de Chaho et FrancisqueMichel. Simplement le dialogue avec la sœur est allongé par les répétitions "d'opéra", très maladroites et inusitées dans la chanson basque ancienne, puis dans le second reproche au père par un nouveau rajout, celui de la citation approximative extraite de la chanson d'Émilie (voir la note 7) : "Père c'est vous qui avez été celui qui m'a vendue, mon frère aîné celui qui a pris l'argent, mon frère puîné celui qui a monté le cheval (ou : qui a passé - sans doute les montagnes - à cheval), mon frère benjamin celui qui m'a aidé". La famille tout à coup s'est agrandie pour retrouver ce vieux thème de rivalité et de complicité familiales de la mal mariée; mais trois vers sur quatre sont faux $(7-5,7-7,7-7,7-6)$, et la maladresse de l'insertion, quant au fond et quant à la forme, est patente. Le correcteur de la version D, tout en bouleversant l'ordre logique et attendu des péripéties, a bien fait de la supprimer. Mais le fait demeure et il est signifiant : les chansonniers (ici Sallaberry ou son informateur) en mal d'inspiration personnelle pour étoffer les complaintes raccourcies par l'oubli au cours du temps, n'hésitaient pas à y recoưdre des "pièces rapportées". Il fallait terminer le chant, c'est évident, par le glas et le deuil étrangement mêlés au "cheval sellé d'or".

La recomposition "logique" des péripéties s'impose ainsi parfois au commentateur, ou, s'il est un peu exigeant, au simple lecteur. L'exemple, beaucoup plus fameux, de la "Chanson de Berterretche" également souletine et qui rapporte sur le mode de la complainte cette fois satirique (contre la fourberie des nobles : ici le comte de Beaumont gouverneur du château de Mauléon pour le roi d'Angleterre, contre les partisans locaux du roi de France, dont Berterretxe, à la fin de la guerre de cent ans vers 1440-50) et tragique (assassinat cu héros de la chanson, angoisses de la mère etc.) pourrait être relu de ce point de vue : il suffirait de déplacer quelques strophes pour donner au récit son "cours" normal, indépendamment des "lacunes", volontaires ou plus probablement d"oubli, qui le rendent largement énigmatique dans le détail. Les complaintes poétiques anciennes, on le sait par d'autres exemples, pouvaient être fort longues, trop longues pour être entièrement retenues au fil des ans et des siècles.

\section{Les problèmes linguistiques}

Ils sont, d'une version à l'autre, et parfois dans la même version, innombrables. On n'en relèvera que quelques-uns, surtout dans l'ordre phonétique et morpho-syntaxique, questions d'orthographe mises à part, de trois types : tributaires du choix dialectal, de l'incorrection pure et simple, des changements intervenus dans l'histoire de la langue ou de type "diachronique".

\section{a) Le choix dialectal.}

La première version de Chaho offre la plus grande régularité, y compris graphique, dans le cadre dialectal souletin. Pour la phonétique, hongariaco a l'avantage, tout en étant conforme à la forme latine et officielle hungaria que Chaho savait (les autres prennent le mot français), de supprimer, comme dans la langue ancienne en tous domaines dialectaux, le groupe muta cum liquida auquel le basque a toujours répugné; mais alors il faut faire la synalèphe hungariacloe) rreguec ou la synérèse hungar(ia) co pour avoir les 7 syllabes du premier hémistiche, les deux solutions étant viables. Pour le reste, galthatu, hountu, heltu etc. la 
phonétique dialectale est régulière, consonnes aspirées comprises. Il faudrait encore relever les variantes biga/miga, arraposta/arrapostu, jaunts/jauntz, berdia/pherdia, algar/elgar/alkhar, guita/quita, erroba/arrauba, etc., de moindre intérêt mais caractéristiques de chaque modèle, le jeu des diphtongues monosyllabiques (en labourdin) et dissyliabiques (en souletin) ayant aussi une incidence sur la métrique.

En morphologie, Chaho est le seul à mettre le vieux futur-potentiel composé conservé en souletin dans hountu diratinian "quand ils seront mûris", qui fait le vers juste et était probablement la forme primitive, alors que les autres pataugent : le futur imperfectif labourdin de F.-M. ontcen direnian "quand ils mûriront" n'a que 6 syllabes, de même que le futur perfectif souletin de Sallaberry huntü direnian d'ailleurs assez incorrect par rapport au bon usage grammatical "moderne"; la version Ozaze... retrouve le mètre en mettant un participe parfait huntürik dinenian, alors que l'on attendrait un participe futur. Sur ce point aussi le texte s'est dégradé après Chaho. Dans les autres formes verbales, Chaho met naizu pour nauzu selon le changement de diphtongue propre au souletin, que reprennent les autres, sauf F.-M. dans le version labourdine : nauçu. Le futur du passé de Chaho ni ezcunturen nintzan "je me serais mariée" (7 syllabes sans synalèphe) reçoit une variante correcte en souletin assez moderne chez Sallaberry bena bai ezkuntüren, tandis que les autres choisissent la version négative qui change le sens du texte (voir ci-dessus) : ez nintçan ezconduren pour F.-M., enündüzün ezkuntüren dans $\mathrm{D}$ avec un allocutif certes très souletin mais qui fausse le vers. Le futur pan-dialectal de Chaho nic ere jauntsiren dut "je vêtirai moi aussi..." conservé par Francisque-Michel est devenu avec altération de l'auxiliaire (mais ce n'est à aucun titre un datif de $\mathrm{l}^{\text {re }}$ personne guipuscoan) jauntsiren dit chez Sallaberry et la version D qui le reprennent dans l'allocutif badizü pour "il y a". Diverses formes s'opposent pour l'impératif, chez Chaho et dans D zuaza "allez" qui exige une synérèse pour la métrique, en labourdin correct F.-M. çoaçi de même; Sallaberry et D utilisent ausși en ordre inversé l'impératif composé ahizpa juan zite portaliala / portaliala ju n zite ahizpa maitia "ma sœur (bien-aimée) allez au portail" (avec ce "portail", la scène est transportée au rez-de-chaussée, autre modification à coup sûr "moderne" de l'histoire). Bien des détails où la réalisation dialectale interfère dans le cours du texte pourraient encore être relevés, parfois contradictoires : Chaho (et peut-être le texte initial ou du moins antérieur) ne met pas le sociatif souletin dans algarrekin, handirekin, qui font rime, évidemment aussi en version labourdine, alors que Sallaberry le met : alkharreki, handireki.

\section{b) Les incorrections grammaticales.}

On peut en relever quelques-unes, y compris d'abord chez Chaho qui écrit un botzez utzi ezazu "abandonnez avec joie" à participe perfectif, là où le second auxiliaire exigerait le radical, ce qu'il fait dans une seconde version écrite en quatrains botzez utz ezazu, formule reprise par les autres, successivement, mais au prix d'un romanisme que Chaho a sans doute voulu éviter : guita çaçu (forme parlée normale, mais faisant 6 syllabes au lieu de 7), kita ezazü. Le ora du premier hémistiche dont Chaho a voulu d'abord faire un vers avec sa rime est évidemment incorrect, et les autres versions ont la seule forme connue de cet adverbe en domaine navarro-souletin orai. Les formes conjuguées de joan sont contradictoires, entre la synérèse parfaitement correcte de l'impératif zuaza (2 syllabes) 
et le participe où la diérèse, d'ailleurs assez traditionnelle, se marque par une aspiration johanen (mais dans la chanson navarraise d'Émilie bihoa ne peut être que monosyllabique ou dissyllabique : voir la note 7), la forme juanen des autres versions étant la prononciation moderne, dissyllabique. L'auxiliaire imperfectif de F.-M. au lieu du perfectif $i c ̧ a n d u$ etc. est redevable du dialecte labourdin.

La version Sallaberry utilise une expression apparemment fautive hanko jente gazteriak, mais il faut sans doute comprendre qu'il y a une juxtaposition "les gens (et) la jeunesse" de toute façon maladroite ( 8 syllabes) et procédant d'une mauvaise compréhension (voir ci-dessus), la version Ozaze... étant revenue aux "petits et grands" hanko txipi handiak qui était à coup sûr la formule originelle. L'étrange "cultisme roman" de F.-M. bere motuz "de son propre mouvement", qui rappelle peut-être de trop près le motu proprio papal, avait dû lui être soufflé par quelque clerc ou latiniste labourdin. Mais il valait mieux à coup sûr, même si le choix des emprunts lexicaux obéit à des motifs personnels et stylistiques, et emprunt pour emprunt, conserver le populaire arrapikatzen (souletin à prothèse initiale plutôt romane : c'était une variante de Chaho) ou errepikatzen (navarrolabourdin), puisque le basque dit "repiquer" pour "répéter" : il ne détonait en rien dans l'ensemble.

\section{c) Les changements diachroniques.}

Un texte oralement transmis est toujours recueilli dans la forme linguistique contemporaine, celle de l'informateur, sauf maintien d'archaïsmes si la langue continue à les admettre, ou, au contraire, s'ils sont incompris (mais en ce cas ils s'altèrent en général et s'éloignant de la forme première deviennent effectivement incompréhensibles). Nous avons donc dans ce texte, et les changements entre la forme la plus "fidèle" apparemment, celle de Chaho, et les suivantes le font bien voir, tout comme dans la "Chanson de Berterretxe" etc. recueillies et écrites à la même époque, une langue de la seconde moitié du XIX siècle, comme dans les chansons médiévales recueillies par Garibay au XVI siècle, un dialecte guipuscoan du $\mathrm{XVI}^{\mathrm{c}}$ siècle à très peu de choses près.

Le basque a beau être une langue qui a peu et lentement changé, c'est dans les derniers temps, à partir du XVIII ${ }^{e}$ siècle où la langue officielle dominante se répand dans la société qu'elle s'est le plus altérée. Ce changement peut surtout être mesuré ici, mais très nettement, dans le traitement de la déclinaison des noms de lieu. Et pour tout dire c'est le nom basque de Tardets Atharratz ou Atharratze qui fait problème et, pour tous les auteurs sauf $\mathrm{D}$, difficulté. Au XIX ${ }^{\circ}$ siècle, et même bien avant en domaine souletin si l'on en croit le "pauvre basque d'Aroue" de Tartas en $1672^{12}$, les noms de lieu à finale consonantique se sont vus adjoindre un -e postiche dit parfois "paragogique" (et pris aujourd'hui, bien à tort, pour une grande spécificité de la langue), qui n'est que la voyelle de liaison indispensable à la déclinaison avec les suffixes à initiale consonantique comme l'inessif indéterminé - $n$ si fréquent pour les noms de lieux (Atharratz, Atharratzen etc.). Donc Chaho commence par Atharratze jaureguian, formule retenue comme titre aussi par Sallaberry et Francisque-Michel (Atharratzeco ezcongaia); mais FrancisqueMichel, qui avait en l'occurrence été bien conseillé, et Sallaberry commencent, en contradiction avec leur titre, par Atharratz jaureg(u)ian. Ils y étaient contraints par l'air : 7 notes sol-sol-si-do-la-sol-mi pour 7 syllabes, et un hémistiche juste par conséquent. C'est que le timbre ou incipit de l'air n'avait sans doute pas changé, ni le support textuel. Mais ensuite, à l'exception de Chaho qui suit l'erreur ini- 
tiale et fait des vers faux (strophe II Atharratze Salala 7 syllabes alors que Atharratz salala serait juste, strophe VI Atharratzeco ceñiac idem), les auteurs ne sont pas conséquents : F.-M. qui a mis correctement encore à la strophe 2 Atharrats (génitif non marqué usuel; sic pour l'orthographe) Salara, écrit à la strophe 6 Atharratceco ezquilec (il ne fait pas de synérèse régulière, donc 8 syllabes au lieu de 7 que ferait Atharrazko ezkilek), Sallaberry de même strophe 2 Atharratzeco hiria ( 8 syllabes au lieu de 7 ) et ainsi de suite.

Bien entendu, la version D, la plus récente et la plus remaniée, n'a pas ce problème : le début est Ozaze Jaurgaiñian, puis Atharratzeko jauna, Atharratze hiria,... Salala, Ozazeko zeñiak, formes régulières en apparence, mais qui ne le sont pas, ce que signale le fait que les hémistiches obtenus sont tantôt justes et tantôt faux. Bref, si la version initiale avait été celle-ci, elle n'aurait pas pu commencer par Ozaze Jaurgaiñian et il aurait fallu sans doute, dans la langue ancienne, Ozazko Jaurgainian, mais comme cette formule à génitif non marqué est peu probable avec des noms de maisons (les exemples médiévaux sont clairs : 1243 Çaro jaureguia etc.), tous les indices concordent (et l'étude de la métrique aussi) pour signifier que c'est une version "collée" sur une chanson précédente, qui devait être le mariage de la fille de la maison noble principale de Tardets (ou d'un autre lieu : voir ci-dessus), et non le mariage d'une fille d'Ossas dans la maison en question. Dans la strophe 2 de Sallaberry et 8 de Jaurgain le mot beste se signale comme postérieur au milieu du XVIII' siècle au lieu de ber(t)ze (voir Annexe I : la lettre de Charles de Luxe de l'extrême fin du XVI ${ }^{\mathrm{e}}$ siècle).

Les couches linguistiques pourraient être cherchées dans d'autres composantes de la chanson: les formes grammaticales des verbes et de la déclinaison peut-être, plus sûrement les emprunts lexicaux (par exemple loratu/doratu...). Mais il serait à coup sûr plus malaisé d'en tirer des conciusions nettes, et le résultat, à savoir le caractère plus ou moins "récent" et altéré d'un texte initial dont les versions connues ne sont que des avatars, n'en serait pas plus clair.

\section{La métrique}

Malgré la tentative incomplète de Chaho de couper les hémistiches et d'en faire des vers, qu'il rectifie dans une autre version, les très nombreuses erreurs métriques qui entachent toutes les versions, certaines (Sallaberry) plus que d'autres, le mètre de cette chanson se définit aisément : vers de 13 syllabes coupé en hémistiches de 7 et 6 en quatrains monorimés ou mono-assonancés dans le style de l'improvisation, l'un des mètres les plus communément utilisés dans la poésie basque. Il faut partir du principe que le texte primitif suivait régulièrement ce mètre, comme du reste l'air, invariable tout au long du récit chanté, le lui imposait. On sait que les poètes anciens, pour peu qu'ils fussent du métier, savaient composer avec justesse : Oyhénart l'observe en 1665 sur quelques exemples de chansons anciennes anonymes à propos de ce qu'il nomme la "quantité" ${ }^{13}$. En plus du mètre et du compte des syllabes, les irrégularités peuvent toucher la rime (ou l'assonance). Certaines irrégularités devaient certes se trouver déjà dans la (ou les) composition(s) originale(s), soit à titre de licences que savaient se permettre consciemment les poètes traditionnels, soit pas insuffisance de métier. Mais, faute de preuves sûres, il est de meilleure méthode de tenir le texte originel supposé inconnu pour conforme à la métrique régulière, même si l'on ignore lar- 
gement quels étaient les principes de composition des auteurs anciens dont les œuvres ont été transmises par tradition orale.

\title{
a) Le mètre et le compte des syllabes.
}

C'est sur ce point que la plupart des textes, généralement antérieurs au XVI ${ }^{e}$ ou tout au moins au XVII ${ }^{e}$ siècle, transmis oralement présentent le plus d'irrégularités. C'est aussi le cas, pour citer encore un exemple connu, de bien des vers des Primitiae de Dechepare pourtant imprimés (1545), et les commentateurs modernes ont bien du mal à retrouver les contractions et prononciations qui permettraient d'accepter que le même mot ait pu avoir trois ou quatre syllabes à l'écrit et deux à l'oral etc. Ce problème se pose évidemment avec acuité pour la "Chanson de Berterretche", où les irrégularités métriques dans ce qui devrait être théoriquement un tercet inégal régulier de 7-7-15 (vers long coupé 8-7 selon l'usage) commencent à la première strophe : 7-8-16 à condition de compter avec élisions et synalèphes :

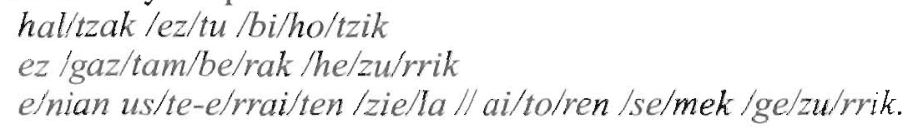

La "correction" du deuxième vers est aisée : il suffit d"enlever le ez initial, pas indispensable surtout en style poétique et vraisemblablement rajouté, pour obtenir le texte régulier; le troisième vers, avec ses verbes dont la forme a pu varier, pose d'autres problèmes : avait-on par exemple le verbe composé erraiten ziela dès le début, et non le simple *zerradala qui fait le vers juste avec la synérèse possible du verbe enian uste, qui lui-même pourrait être avantageusement remplacé par *enakian etc.? Mais le second hémistiche est plus difficile à retoucher : toutefois *aitorseme pourrait avoir été la composition primitive (non attestée dans la langue historique) "fils de bon père" (= "noble") selon un schéma bien connu en basque.

Plusieurs rétablissements de cet ordre sont suggérés par le texte lui-même dans Atharratz(e) Jauregian.

$1^{\circ}$ Dans la première version de Chaho remise en forme de quatrains, mais que Chaho avait sans doute cru d'abord plus ou moins clairement huitain d'octosyllabes et heptasyllabes alternés (d'où son ora du premier hémistiche strophe V pris pour vers etc.), le rétablissement peut se faire en additionnant ou en supprimant une syllabe, au besoin en changeant un mot, parfois en prenant en compte diérèses, synérèses ou synalèphes, à condition toutefois de le faire systématiquement, ce qui peut créer des difficultés. Les termes modifiés sont mis ici entre barres, les faits de synérèses et synalèphes entre parenthèses chaque parenthèse formant une syllabe unique pour les seuls vers métriquement irréguliers :

\author{
Strophe I \\ Santa Clara \\ Atharratz jaureguian bi citroiñ loratu; \\ Hongariac(o e)rreguec batño du galthatu; \\ Arrapostu(/a/ u)khen du eztirela hountu etc... \\ Strophe II \\ Aita /zuk/ saldu naizu biga bat bezala \\ Bai eta des/t/erratu* Hongar(ian) behera;
}


Ama biz(i u)khen banu...

Ezcunturen /ninduzun/ Atharratz Salala.

(*NB : au lieu du deserratu original il suffirait de deserritu pour faire un assez bon équivalent basque de l'hispanisme desterratu.)

Strophe III :

... larrauba/ berdia (en souletin la diérèse -di/a est régulière)

Botzez hutziko duzu/ (ou : lutz dezazula/)...

Strophe IV : la strophe est juste, sauf pour l'absence de synalèphe ou élision dans zure alhaba, et donc vraisemblablement, selon les principes exposés ici, peu altérée par rapport à la version originale.

Strophe V :

Ahizpa zoaz (avec diérèse) ora/i/ (ou : zoaz(a o)rai)... Tout le reste de la strophe est juste (à heguoua la diérèse souletine est aidée par la graphie).

Strophe VI, rectification aux premier et dernier hémistiches :

Atharratzco çeñiak...

... beltzez dira /janzten/. Le verbe janzte "(se) vêtir" s'impose d'autant plus aisément qu'il est déjà répété dans le dialogue des deux sœurs.

$2^{\circ}$ Dans les autres versions, celle de Francisque-Michel peut être mise de côté, parce qu'elle est une labourdinisation nécessairement altérée par rapport au texte souletin, ce que le commentaire de l'auteur souligne llui-même avec raison pour senhar gueia, d'ailleurs en phonétique plutôt souletine pour gueia.

Les remplissages à répétitions de la version Sallaberry sont si maladroits aux strophes 4 et 5, et même 6 (retour de ingoiti horra dïzü qui a plu sans doute à cause de sa bonne formulation souletine), qu'il est vain d'y chercher une forme primitive plus ou moins correcte. La strophe 2 qui décrit Tardets, dont il y a de bonnes raisons de penser (absence chez Chaho notamment : voir ci-dessus) qu'elle est postiche, se prête pourtant à peu près à un rétablissement complet :

premier vers : Atharrazko hiria/da/ hiri ordoki

deuxième vers :... /alderdi/bateti

troisième vers : /bigarren/ aldeti.

\section{b) La rime et l'assonance.}

Le quatrain monorime d'improvisation, rime-assonance -tu (trois fois) et $-d u$ (une fois) à la strophe I, rime -ala ou assonance - $a$ (variante behera, meilleure techniquement qu'une répétition de trois bezala : notons que c'est la strophe du mariage-vente, qui passe d'une chanson à l'autre depuis $A n d r$ 'Emili) à la strophe II, rime -ia à la strophe III, assonance - $i$ - avec -kin et -rik à la strophe IV (elle est assez banale en poésie improvisée, mais la forme souletine de sociatif - $k i$ que Chaho évite d'abord la renfoncerait en donnant la structure phonétique inversée $k i /$-ik...), rime -en à la strophe VI (où le romanisme phartitzen serait avantageusement remplacé par/da joaiten/ qui renforcerait le parallélisme avec la fin du premier vers : du joiten...). Cette régularité très acceptable dans la poésie improvisée ancienne assonancée est rompue toutefois à la strophe $\mathrm{V}$ qui fonctionne sur deux rimes : -ra aux deux premiers vers, -(r)ri aux deux derniers (l'équivalence des deux vibrantes peut être admise comme licence régulière, comme l'a été toujours celle des sifflantes d'articulation apicale et dorsale). Comme il n'y a aucun 
moyen aisé de modifier, il faut penser qu'elle était dans la version primitive, car c'est sans doute la strophe capitale, celle où se dessine, et change selon le vent, le destin de la fiancée, et la rupture sonore pouvait alors symboliser la rupture tragique de la vie. Par ailleurs la strophe est métriquement juste, et fortement harmonisée par les sonorités vocaliques opposées $-i-a-a-a-i-a-a-i-a-i-a-a-i$ etc. qui se répondent à l'intérieur des vers et des hémistiches : ipharra balinbadal goraintzi Salari/ Et'aldiz ene tcherca/ Jin dadila sarri).

Dans les versions longues ou "allongées", ce qui se remarque surtout c'est la répétition des mêmes mots à la rime, que la poétique un peu exigeante, même improvisée, récuse en principe : dans la version Salaberry str. 3 bezala deux rimes suivies, str. 4 nizala deux rimes suivies, str.5 zirela deux rimes suivies.

\section{Conclusion}

Les quatre versions principales de la chanson communément intitulée Atharratze jaureguian posent de redoutables difficultés à qui veut la considérer de près, et ceci dans tous ses éléments : sujet, développement du récit poétique, réalisation linguistique et métrique. A mesure que les versions viennent s'ajouter les unes aux autres (et rien ne dit à cet égard que l'aventure du texte est aujourd'hui achevée), les difficultés, allant jusqu'aux contradictions formelles dans le même texte ou d'un texte à l'autre, ne font qu'augmenter. Tout ceci rend probable le fait suivant : la version initiale, celle de Chaho, morceau rescapé et déjà tronqué d'une ancienne complainte vraisemblablement médiévale, l'une des versions locales de la chanson de la "mal mariée", a été détournée de sa primitive histoire légendaire pour "coller", tant bien que mal et plutôt mal que bien, au second mariage du seigneur de Tardets-Luxe dit "Charles de Luxe" dans la seconde moitié du $\mathrm{XVI}^{\circ}$ siècle.

Dans cette histoire aussi confuse que longue, jusqu'aux collectes de la seconde moitié du XIX ${ }^{\mathrm{e}}$ siècle où le texte est pour la première fois sans doute fixé par l'écrit, il a subi toutes sortes d'altérations, au plan des faits relatés et des personnages, du texte et de la langue, et bien entendu de la forme poétique. La chanson souletine Atharratze jauregian représente un cas particulier de ce qu'il est advenu des compositions poétiques anciennes transmises par tradition orale : car, audelà des particularités, elle illustre et éclaire aussi le cas général, et offre bien des points communs avec le traitement subi par tous les autres textes de même origine. La grille d'analyse textuelle qui s'impose ici est bien différente, pour plusieurs points essentiels et même si tout texte est soumis au fils du temps à l'altération, de celle de la littérature écrite et transmise par l'écrit.

Jean-Baptiste ORPUSTAN

Professeur de langue et littérature basques Université Michel de Montaigne - Bordeaux III 


\section{NOTES}

1. Dans un article intitulé "Le Roman d'Arles (Aix-en-Provence, Musée Arbaud, M.O.63, fo30-50v"): problèmes d'une édition critique", H.-Ch. Haupt étudie les altérations subies, pour la métrique en particulier, par une compilation manuscrite de pièces occitanes en vers copièe entre 1373 et 1376, Actes du IV Congrès International de l'AEIO, Vitoria-Gasteiz 22-28 août 1993, Vitoria-Gasteiz 1994, Tome I p. 127-138.

2. Parmi les notables bordelais d'origine basque au début du XVIe siècle, on compte notamment Jean d'Ibarrola, originaire de Sare comme l'avocat Bernard Lehet, docteur en droit civil et droit canon, Recteur de l'Université de Bordeaux en 1521, décédé en 1537.

3. Le recueil d'A. Zavala intitulé Euskal erromantzeak, Romancero vasco (Auzpoa, Zarauz 1998 p. 38 60) relève 19 publications différentes de cette chanson, la première étant selon Francisque-Michel le récit en français relevé par "M. de Quatrefages" auprès d'une Biarrotte. Parmi les commentaires basques, outre celui de l'auteır lui-même qui est le dernier en date, il faut citer ceux des ouvrages suivants: J. Lakarra, K. Biguri, B. Urgell Euskal Baladak Tome II, Saint-Sébastien 1983, p. 72-79; L. M. Mujika Euskal lirika tradizionala, Tome II, Saint-Sébastien 1984.

4. Francirque-Michel, Le Pays basque, sa population, sa langue etc. Paris 1857, p. 263-266.

5. Jean-Dosininique-Jules Sallaberry, Chants populaires du Pays basque, Bayonne 1871, p. 283-287.

6. La version Ozaze Jaurgaiñian apparaît pour la première dans le livre de J. de Jaurgain, La tradition au Pays basque, Bayonne 1899, à la rubrique intitulée Quelques légendes poétiques du Pays de Soule, l'auteur s'efforçant, assez maladroitement, de justifier ses explications par "toutes les versions que j'ai recueillies moi-même en Soule et Basse-Navarre"; mais il était bien tard, sans doute, pour y trouver la "bonne" version. Elle est reproduite sous le titre Atharratzeko ezkongaia dans l'anthologie poétique des écrivains basques de France de K. Etxenagusia, Iparraldeko euskal idazleak, Bilbao 1981, p. 15-16.

7. J. de Jaurgain, La Vasconie, $2^{2}$ partie, p. 70-76. Les seigneurs de Tardets eux-mêmes étaient aussi seigneurs d'Ahaxe (il reste encore les ruines de la tour de leur château fort) par le mariage de l'héritière d'Ahaxe Arnaude avec Guillaume-Arnaud seigneur de Tardets au milieu du XIII ${ }^{e}$ siècle : ibidem, p. 254.

8. La lettre basque que Charles de Luxe-Tardets dicte ou fait écrire à l'une de ses filles est reproduite avec la correspondance basque de la dame d'Urtubie (qui devait être Aimée de Monréal d'Urtubie qui avait épousé le 17 septembre 1574 Jean d'Alzate d'Urtubie son cousin) de la même époque pour les mêmes raísons d'espionnage au profit du roi d'Espagne, par José Manuel Florestán Imizcoz dans Fontes Linguae Vasconum, año XXV, ${ }^{\circ}$ 63, mayo-agosto, Pamplona 1993, "Conflictos fronterizos, espionaje y vascuence a finales del siglo XVI : 20 documentos ineditos", p. 177-219. Cette lettre avec sa traduction française est reproduite en Annexe I au présent article.

9. Les liens entre les seigneurs pyrénéens et la monarchie hongroise sont historiques mais lointains: dans les Actes du IV Congrès International de l'AEIO... signalés ci-dessus dans la note 1, p. I13-125, un article de R. Harvey "Les contexte des "performances" des troubadours" se réfère au mariage de Constance d'Aragon avec le roi Emeric II de Hongrie en 1198 : le troubadour Peire Vidal aurait suivi la princesse aragonaise, et le troubadour Gaucelm Faidit aurait séjourné déjà lui aussi en Hongrie pour le mariage de Marguerite de France sceur de Philippe-Auguste avec Béla III en 1186. En tout cas la référence à la Hongrie est dans la littérature occitane depuis ce temps-là, et il est assez probable que c'est le point de départ d'une thématique des "mariages hongrois" qui serait passée aussi dans la poésie basque médiévale.

10. Trois strophes, la seconde tronquée, de ce chant médiéval en tercet inégal remontant pour son thème au temps où se faisaient des mariages entre familles seigneuriales navarraises et "maures" d'Al Andalus, sont rapportées par Oyhénart dans sa lettre dite de L'art poétique basque (1665), Gure Herria 1967 p. 220. La troisième comme la précédente fait parler la jeune "mal mariée":

Aita nuen salçale

Ama diru harçale

Anaveric chipiena mairuetaric guençale

"Mon père fut le vendeur, ma mère celle qui prit l'argent, le plus petit de mes frères celui qui m'arracha aux Maures".

11. Paris S.E.C., 1975, p. 929. Il y a aussi un "Vicomte de Sainte-Claire" à Madrid au début du XVII . siècle. Tallemant des Réaux, Historiettes, Tome I, p. 965. Bibliothèque de La Pléiade.

12. Dans les noms de sa dédicace basque de 1666 au marquis de Monein, Tartas aligne, parmi bien des approximations phonétiques dans les noms de lieux, les voyelles finales après consonne: Barcoiçé, Domicigné, Lohitçuné, Amenduçé, Larçeualé..., mais par toujours : Mitrigna (qui est la prononciation d'Aroue pour ce qui est dans toute la Basse-Navarre "Bithirine" et sans doute anciennement "Bithirina" avec le -a final de l'étymon latin vitrina), Ispoura, Sarhia, Çaldia...

13. Voir ci-dessus la note 10. 


\section{Annexe I : la lettre de Charles de Luxe de 1595, écrite par sa fille.}

N.B. La transcription, avec malheureusement diverses modifications graphiques, ponctuation etc., alors que ces textes demanderaient à être reproduits et publiés d'abord texto, sans la moindre modification, est de l'auteur de l'article signalé à la note 7 ; puisque le mal est fait, nous y avons ajouté les cédilles souvent omises dans l'écriture ancienne, faute de quoi le texte a pu être pris à contresens. La note de réception, en espagnol, précise : "Ce soir j'ai reçu la présente du seigneur de Lussa, et comme elle est écrite en basque qu'écrit une de ses filles, j'envoie ici ce qu'il m'y dit'. La question à poser est celle-ci : pourquoi Charles de Luxe fait-il écrire cette lettre à sa fille? pour ne savoir écrire ou ne le plus pouvoir? par précaution? En tout cas la langue est dans l'ensemble de fort bonne qualité, aux habitudes orthographiques du temps près (pas de double $r$ r etc.).

Jauna : eremerçiazen derauçut çegei çoure merchediac hainberçe couidado baitu niçaç. Heben beri bazu ditugu, hamenz niri particularci adiscide batec egori deraut erege Franciacouac eman duiela batailla duce de Umenanaren eta espainolen countre, erege çauri dela eta mous de Anbila Franciaco Counestabliaren anaie baita houra hil, () haniz bi aldetaric, hamasei mila, baratu dituçu campouan. Barbera Bordelera baita gindenian çoure merchedia abisaturen dut, (;) halaber suplicazen nizauçu auçtore pareba(t) nahi derautaçun chercatu neure duhuruian. Charles de Lusse.

Mous de Guisa eta Agaramoundeco jauna hil dira haniz berçe prinçipalci, erege (il faudrait l'ergatif : erregec) irabaçi canp bataila. Baionara dira Bordeleco counseillerac, urcagei dute hango bord (peut-être pour "Bordeleco") tradiçionian cousentac, haniz dela berçeric ere dioite. Letra haur esciribatuç geroç ençun dut duc de Umena ere hil dela.

Traduction:

"Monsieur, je vous remercie pour ce que votre grâce prend tant de soin de moi. Nous avons ici quelques nouvelles, du moins un ami m"a envoyé dire en particulier que le roi de France a livré une bataille contre le duc de Mayenne et les Espagnols, que le roi est blessé et monsieur d'Anville (Damville) qui est frère du Connétable de France, lui est mort; beaucoup des deux côtés, seize mille, sont restés sur le champ de bataille. Comme le barbier (ou : Barbier) est à Bordeaux, j'aviserai votre grâce quand il reviendra; de même je vous supplie de chercher pour moi une paire d'autours (oiseaux de chasse), que je paierai de mon argent. Charles de Luxe.

Monsieur de Guise et le seigneur de Gramont sont morts avec beaucoup d'autres principaux; le roi a gagné sur le champ de bataille. Les conseillers de Bordeaux sont venus à Bayonne; ceux de Bordeaux qui ont consenti à la trahison sont promis à la potence, et ils disent qu'il y en beaucoup d'autres. Depuis que cette lettre a été écrite j'ai entendu dire que le duc de Mayenne aussi est mort."

\section{Annexe II : Atharratz jauregian avec métrique rectifiée.}

A partir des ajustements présentés dans le commentaire, voici comment la chanson pourrait être proposée, dans sa version ou les restes de sa version la plus ancienne, en excluant la référence à la fille d"Ossas et les strophes répétitives ou importées de la chanson navarraise de "Dame Émilie". Les péripéties absentes de 
la chanson, qui auraient pu ou même dû y figurer à l'origine, sont signalées entre parenthèses et par des points de suspension. Graphie basque moderne mais sans modification phonétique, synalèphes marquées entre parenthèses.

I

Atharratz jauregian bi zitroiñ loratü;

Hungariak(o e)rregek batño dü galthatü.

Arrapostü(a ü)khen dü eztirela huntüu,

Huntü diratinian batño ukhenen dü.

II

Atharrazko hiria da hiri orloki :

Ur handi bat badizü alderdi bateti,

Erregeren bidia badoa erditi,

Maria Madalena bigarren aldeti.

... (Plusieurs années après, le jour du départ pour le mariage ou pour la Hongrie :)

III (Santa-Klarak aitari :)

"- Aita zük saldü naizü biga bat bezala,

Bai eta deserritü Hongarian hara;

Ama biz(i ï)khen banü aita zü bezala.

Ezkunturen nintzan ni Atharratz Salala."

IV (Ahizpak Santa-Klarari :)

“- Ahizpa jauntz ezazü arrauba berdia,

Nik ere jauntziren düt satina xuria;

Ingoiti hor heliü da zure senhar geia.

Botzez ütziko düzï zure sorth-etxia."

... (La conversation entre les deux sœurs a pu se poursuivre : voir les strophes 4 et 5 de Sallaberry, avec la visite au "portail", malheureusement trop incorrectes et répétitives pour être rétablies, et le orai itératif de $\mathrm{V}$ :)

V (Santa-Klarak ahizpari :)

"- Ahizpa zuaz(a o)rai salako leihora

Iphar ala hegua zer den jakitera;

Ipharra balinbada, goraintzi Salari :

Et' aldiz ene txerka jin dadila sarri."

VI (Santa-Klarak aitari :)

"- Aita johanen gira orai elgarreki;

Etxerat jinen zira xangrin handireki,

Bihotza tristetüric begiak büstirik

Bai eta zur(e a)lhaba thumban ezarririk."

... (Les incidents survenus après cette menace devaient être évoqués ici par le chant ou, peut-être, récités. Le départ est assimilé à un jour d'enterrement :)

VII

Atharrazko zeñiak ber-berak dü joiten,

Andere Santa Klara herritik da joaiten,

Haren peko zaldia urhez da zelatzen,

Hanko txipi handiak beltzez dira janzten. 